\title{
COMPUTING AN OPERATING STRATEGY FOR AN ACTIVE BODY CONTROL WITH DYNAMIC PROGRAMMING IN THE AUTOMOTIVE AREA
}

\author{
Marlene Utz, Phillipp Hedrich, and Peter F. Pelz
}

Technische Universität Darmstadt, Chair of Fluid Systems

Otto-Berndt-Str. 2, 64287 Darmstadt, Germany

e-mail: \{marlene.utz, phillipp.hedrich, peter.pelz $\} @$ fst.tu-darmstadt.de

Keywords: Active Body Control, Dynamic Programming, Operating Strategy, Skyhook

\begin{abstract}
Customers expect a modern automobile to be comfortable and safe. The spring damper system in the wheel suspension is the most important component to ensure both. During the design process of the spring damping a physically based conflict between these two main functions arises. For passive systems improving the driving comfort will always worsen the driving safety and vice versa. We developed an active air spring damper system (AASD) which enables us to increase the driving comfort while keeping driving safety constant. Since auxiliary power is necessary for the adaptive control a new challenge is to handle the limited energy supply. In this paper we present a primal heuristic for the determination of an operating strategy for the AASD for a given route and a given limited energy supply. We appraise feasible solutions and present runtime benchmarks.
\end{abstract}




\section{INTRODUCTION}

Automobiles are equipped with spring damper systems to ensure the safety and to allow a comfortable ride. Currently, most spring damper systems work passively. When designing a passive spring damper system one is always confronted by the conflict between driving safety and driving comfort. At a certain point it is not possible to improve the safety without simultaneously worsening the comfort and vice versa [1]. To further increase the driving comfort for a constant driving safety, active spring damper systems have to be used. Within the Collaborative Research Centre 805 "Control of Uncertainty in Load-Carrying Structures in Mechanical Engineering" an active air spring damper (AASD) was developed at the Chair of Fluid Systems at Technische Universität Darmstadt. The working principle is based on the adaptive control of the axial force by changing the effective area of the AASD dynamically [2]. Since auxiliary power is necessary for the adaptive control of the axial force, another challenge is to handle limited energy supply. In this paper we present a primal heuristic for the determination of an operating strategy for the AASD for a given route and a given energy supply. The route consists of sections distinguished by the corresponding uncertain road condition. The uncertain road condition is represented by an uncertainty set with scenarios. The working principle and the input data of the optimization are described in Section 2 of this paper.

The goal is to find an operating strategy with maximal comfort, while keeping a level of safeness. Solving the problem in a reasonable timespan requires an efficient algorithm. The specific structure allows the usage of a dynamic program (DP), which is presented in the second part of the paper. The dynamic program, decides how much energy is allowed to be used on a single route section. The steps of the DP are the sections of the route and the considered states are the remaining energy supplies before entering the section. The DP finds the optimal solution for a given class of problems. Applying DP to solve this problem requires discretization of the energy supply. In order to relate an increase of demanded energy to an increase of comfort we modify the DP: Increasing the number of considered initial steps and reusing the calculated interim results enables us to compute operating strategies for a large number of energy supplies simultaneously.

Furthermore we give runtime benchmarks for the modified version of the DP. We increase the number of route sections and the number of considered states in every step of the DP. We present solution quality benchmarks and analyse a solution.

\section{TECHNICAL DESCRIPTION}

The requirements of an spring damper system are among others to guide the wheel safely to reach a good driving comfort and driving safety. When investigating a passive spring damper system in a quarter-car vehicle (cf. Fig 1a) we deal with a two degree-of-freedom-system, whose operating point is predefined by the spring stiffness and the damper constant. The operating point is fix if the spring damper system is not adjustable. When designing such systems a trade-off between driving comfort and driving safety has to be made. The standard deviation of the body-acceleration is used to rate the driving comfort and the standard deviation of wheel load fluctuation is equivalent to the driving safety [1]. A small value of the the standard deviation of the body-acceleration implies high driving comfort. The conflict between driving comfort and driving safety is illustrated in the conflict diagram in Figure $1 \mathrm{~b}$. The Pareto-front shows the limitation of a passive spring damper system.

In order to overstep the limitation of the passive system an active system which applies forces during usage is used. Within the Collaborative Research Centre 805 - "Control of Uncertainty 


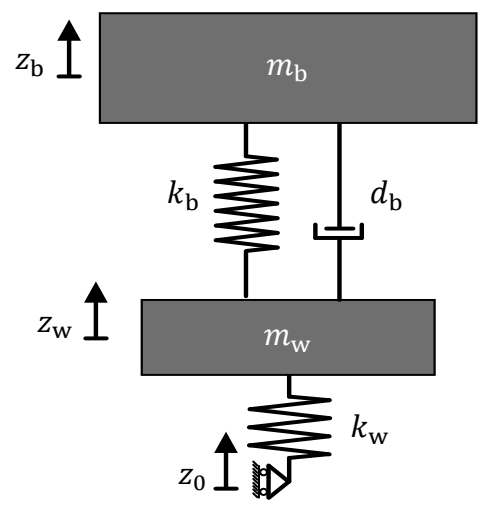

(a)

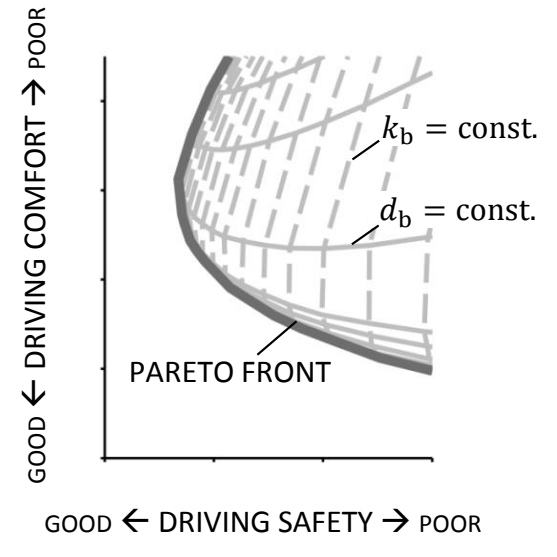

(b)

Figure 1: Conventional topology of a vehicle suspension (a) and its limitation for vertical dynamics by a pareto front (b) [2].

in Load-Carrying Structures in Mechanical Engineering" an active air spring damper (AASD) was developed (cf. Fig 2) [2], which combines the advantages of an air spring damper system and an active system [3] . By changing the load carrying area of the air spring during the usage the axial force

$$
\left.F(t)=A(t)\left[p(t)-p_{a}\right)\right], \text { with } A(t)=A_{1}(t)-A_{2}(t)
$$

can be alerted.

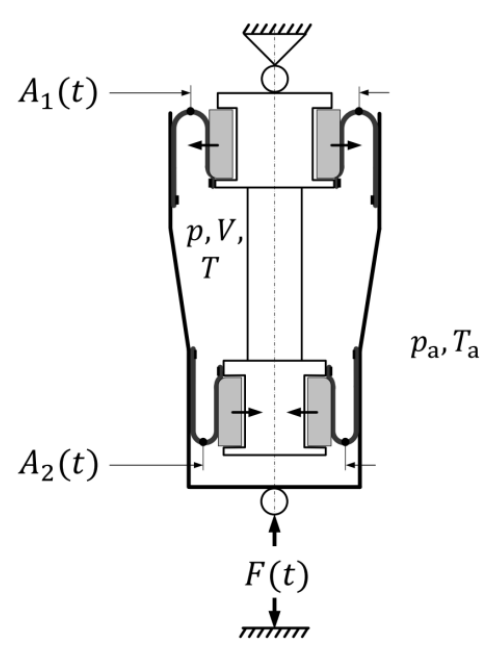

Figure 2: Schematic diagram of the double bellows active air spring. The axial force is $F(t)=$ $\left.A(t)\left[p(t)-p_{a}\right)\right]$, with the load-carrying area $A(t)=A_{1}(t)-A_{2}(t)[2]$.

The AADS allows to improve the driving comfort at almost the same level of safety. For this reason the driving comfort is the objective of the optimization while keeping the safety level constant.

Without taking the random uneveness of the road into account the road excitation can be treated as a stochastic excitation [4]. The power spectral density (psd) of the road profile con- 
tains informations about the unevenness of the road and is given by

$$
\Phi_{h}(\Omega)=\lim _{X \rightarrow \infty} \frac{4 \pi}{X}[\hat{h}(\Omega)]^{2},
$$

with $\hat{h}(\Omega)$ is the amplitude spectrum of the road profile and $X$ the period length. The psd is approximated by

$$
\Phi_{h}(\Omega)=\Phi_{h}\left(\Omega_{0}\right)\left[\frac{\Omega_{0}}{\Omega}\right]^{-\omega}
$$

where $\Omega=2 \pi / L$ denotes the angular wavenumber and $\Phi_{0}=\Phi_{h}\left(\Omega_{0}\right)$ describes the value of the psd at the reference angular wavenumber $\Omega_{0}=1 \mathrm{~m}^{-1}$. The road condition is characterized by $\Phi_{0}$ and $\omega=v \Omega$ where $v$ is the driving velocity. Often the condition of the roads and the driving velocity are uncertain. In order to take this fact into account sets $\mathrm{Sc}$ of all occurring triples $\left(\Phi_{0}, \omega, v\right)$ are used. The given route is divided into route sections $i=1 \ldots N$ with homogeneous road conditions. A road with the homogeneous condition can be divided into more than one route section. One calls these triple a section-scenario $\mathrm{sc}_{i}$ and a sequence $\mathrm{sc}_{1}, \ldots, \mathrm{sc}_{N}$ of section-scenarios corresponding with the sections of the consider route route-scenario $\mathrm{sc}_{r}$. For every section-scenario a probability is given. We assume that the road condition get deterministic in the moment the vehicle enters the route section. One can generate information by a pre-scan of the road.

To use an active system it is necessary to implement a controller, which process signals provided by sensors of the vehicle to calculate the required active force. The optimization chooses from a set of controllers. A possible control strategy is a Skyhook control which is based on the assumption that the driving comfort is optimal when the oscillating body of the vehicle is damped with respect to the horizon. This strategy simulates the responses that would occur if a damper was installed between the vehicles body and the horizon [3]. The required active force between wheel and body is given by [5]

$$
F_{\text {req }}(t)=d \dot{z}_{b}(t) \text {. }
$$

The optimization chooses from a set of damping constants $d \in D$.

Given a model of the AADS, a route section with a triple $\mathrm{sc}=\left(\Phi_{0}, \omega, v\right)$ that characterizes the road condition and a Skyhook controller with damping constant $d$ one calculates the corresponding values of the standard deviation of the body-acceleration $\sigma\left(\ddot{z}_{b}(d, \mathrm{sc})\right)$, the standard deviation of the wheel load fluctuation $\sigma\left(F_{w}(d, \mathrm{sc})\right)$ and the mean power input $\bar{P}(d, \mathrm{sc})$. In order to ensure a certain level of safety only those damping constants $d \in D$ are chosen for a section with certain road conditions whose $\sigma\left(F_{w}(d, \mathrm{sc})\right)$ is lower than a given bound.

If the length $l_{i}$ of the route section is given one calculates the total energy consumption $e_{i}(d, \mathrm{sc})$ for the section $i$ via

$$
e_{i}(d, \mathrm{sc})=\bar{P}(d, \mathrm{sc}) \frac{v}{l_{i}} .
$$

\section{DYNAMIC PROGRAMMING}

The optimization problem is to find a partition of the available energy $e_{r}$ to the route section such that the resulting comfort is maximal and a minimum level of driving safety is guaranteed. Since the selection of the controller determines the energy usage for given section-scenarios one 
has to find a sequence of controllers for every road-scenario which respects the energy partition. Due to the complexity of the problem and the vast amount of controller-sequences which need to be calculated, it is necessary to employ a efficient algorithm to solve the problem in reasonable time. The structure of the problem allows one to formulate a Dynamic Program (DP). DPs are used to solve problems which consist of a sequence of depending sub-problems. The DP starts with solving the smallest sub-problems and proceeds to solve the next bigger sub-problems by reusing the solution of the smaller sub-problems. This strategy leads to an optimal solution of the original problem [6]. It is possible to formulate a optimization problem containing a sequence of depending sub-problems as a DP if the optimal solution of a sub-problem contains the optimal solution of the next smaller sub-problem.

If a problem is represented by the following it can be solved with DP resp. algorithm 1 [6].

$$
\begin{array}{rlrl}
\min F\left(x_{1}, \cdots, x_{n}\right) & =\sum_{k=1}^{n} f_{k}\left(z_{k-1}, x_{k}\right) & \\
\text { s.t. } z_{k} & =\operatorname{tr}_{k}\left(z_{k-1}, z_{k}\right) & & \forall k=1 \ldots n \\
z_{0} & =a & \\
z_{n} & =b & \\
z_{k} & \in Z_{k} & \forall k=1 \ldots n \\
x_{k} & \in X_{k}\left(z_{k-1}\right) & \forall k=1 \ldots n
\end{array}
$$

whereby the terms are defined as follows:

$$
\begin{array}{ll}
n & \text { number of decision steps, specifies how many decisions have to be taken. } \\
z_{k} & \text { state variable, specifies the state of the system after decision step } k . \\
Z_{k} & \text { state set: set of all possible states of the system after decision step } k . \\
z_{0}=a & \text { given initial state of the system. } \\
z_{n}=b & \begin{array}{l}
\text { given } \text { end state of the system. } \\
x_{k}
\end{array} \\
X_{k}\left(z_{k-1}\right) & \begin{array}{l}
\text { decision variable, specifies the decision which is made in step } k \\
\text { decision set of all possible decisions which can be made in step } k, \text { if }
\end{array} \\
\operatorname{tr}_{k}\left(z_{k-1}, z_{k}\right) & \begin{array}{l}
\text { transfer function, specifies the state } z_{k} \text { of the system after taking decision } \\
z_{k} \text { starting from state } z_{k-1} .
\end{array} \\
f_{k}\left(z_{k-1}, x_{k}\right) & \begin{array}{l}
\text { evaluation function of the step, which specifies the effect of decision } x_{k} \text { of } \\
\text { the objective function starting from state } z_{k-1} . \text { It is necessary that } f_{k} \text { is only } \\
\text { depending on } z_{k-1} \text { and } x_{k} .
\end{array}
\end{array}
$$

One calls a sequence of decisions $\left(x_{h}, x_{h+1}, \ldots, x_{l}\right)$ which transfer the system from state $z_{h-1}$ into $z_{l}$ a policy. A policy $\left(x_{h}^{*}, x_{h+1}^{*}, \ldots, x_{l}^{*}\right)$ which minimizes $\sum_{i=h}^{l} f_{i}\left(z_{i-1}, x_{i}\right)$ is called optimal policy. Let $P_{k}\left(z_{k}\right)$ be the problem of finding the optimal policy which transfers the system from state $z_{k}$ into $z_{n}$ and $F_{k}^{*}\left(z_{k}\right)$ the objective function of this problem. The fact that the evaluation function $f_{k}\left(z_{k-1}, x_{k}\right)$ only depends on $z_{k-1}$ and $x_{k}$ leads to the optimality principle of Bellman which says that if $\left(x_{h}^{*}, \ldots, x_{l}^{*}\right)$ is an optimal policy to transfer the system from $z_{h-1}$ into $z_{l}$ and the policy leads to a state $z_{j}^{*}(h-1 \leq j \leq l),\left(x_{h}^{*}, \ldots, x_{j+1}^{*}\right)$ and $\left(x_{j+1}^{*}, \ldots, x_{l}^{*}\right)$ are 
optimal policies to transfer the system from $z_{h-1}$ into $z_{j}^{*}$ resp. $z_{j}^{*}$ into $z_{l}$. Using this principle leads to:

$$
F_{k-1}^{*}\left(z_{k-1}\right)=\min _{x_{k} \in X_{k}\left(z_{k-1}\right)}\left\{f_{k}\left(z_{k-1}, x_{k}\right)+F_{k}^{*}\left(z_{k}=t_{k}\left(z_{k-1}, x_{k}\right)\right)\right\}
$$

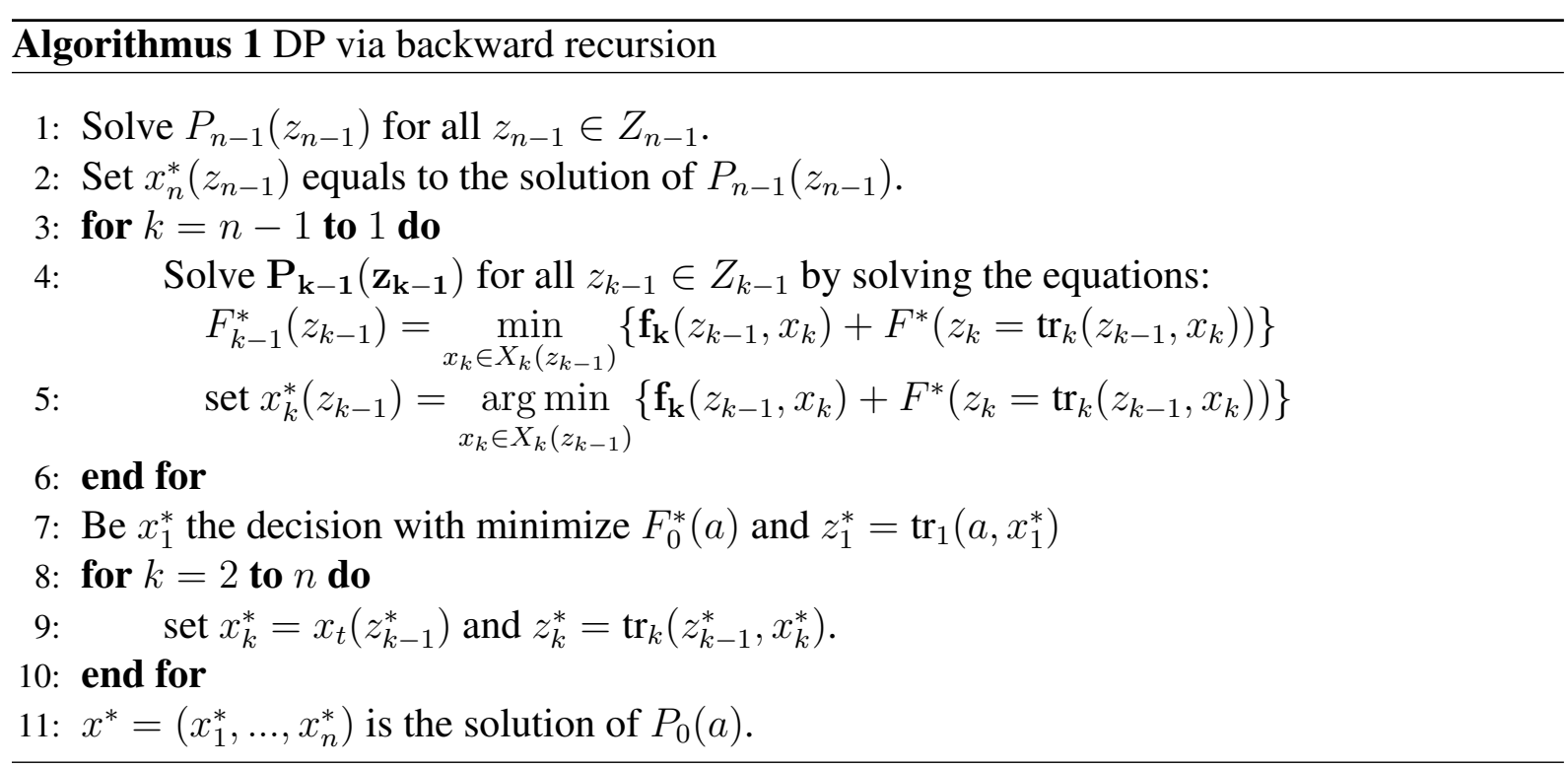

In the developed DP the sections $i=1 \cdots N$ of the routes are the decision steps and the decision $x_{i}$ which is taken is how much energy is maximally consumed on the section. In case of a Skyhook controller one calculates the best damping constant $d$ for a given $x_{i}$ and occurring $\mathrm{sc}_{i}$ by solving

$$
d_{i}\left(x_{i}, \mathbf{s c}_{i}\right)=\max \left\{d \in D: e_{i}\left(d, \mathbf{s c}_{i}\right) \leq x_{i}\right\} \quad \forall i=1 \ldots N .
$$

The states $z_{i}$ of the DP are the remaining energy supply after section $s_{i}$. Thus, the initial state $z_{0}=e_{r}$ is the energy supply for the entire route. There is no requirement for the energy supply at the end of the route. As you can exchange energy for comfort it is advisable to set $z_{N}=0$. There is always one controller which causes the highest power input on a route section. It is impossible to use more energy on this route section. Let $e_{i, \max }$ be this energy and

$$
\hat{e}_{i, \max }=\sum_{l=i}^{N} e_{l, \max } \quad \forall i=1 \ldots N
$$

be the maximum amount of energy which can be consumed starting from section $i$ till the end of the route.

To get the state sets $Z_{i}$ we choose an energy-step $E$ and generate states $z_{i}=n E$ (starting with $n=0$ ) by increasing $n$ by one as long as $n E \leq z_{0}$. We insert this states $z_{i}$ into $Z_{i}$. This leads to

$$
Z_{i}=\left\{z_{i}=n E: n E \leq z_{0}, n \in \mathbf{N}\right\} \quad \forall i=0 \ldots N
$$

In order to relate an increase of demanded energy to an increase of comfort we modify our DP. The initial state $z_{0}$ is substituted by a set of $Z_{0}$ initial states. To get the state sets $Z_{i}$ we 
choose an energy-step $E$ and generate states $z_{i}=n E$ (starting with $n=0$ ) by increasing $n$ by one as long as $E n \leq \hat{e}_{i, \max }$. We insert this states $z_{i}$ into $Z_{i}$. This procedure leads to

$$
Z_{i}=\left\{z_{i}=n E: n E \leq \hat{e}_{i, \max }, n \in \mathbf{N}\right\} \quad \forall i=0 \ldots N .
$$

The transfer function is given by

$$
\operatorname{tr}_{i}\left(z_{i-1}, x_{i}\right)=z_{i-1}-x_{i} \quad \forall i=1 \ldots N .
$$

To formulate a DP it is furthermore needed to define an evaluation function for every decision which can be made. Since the objective is the driving comfort and the comfort is measured by the standard deivation of the body acceleration $\sigma\left(\ddot{z}_{b}\right)$, this value is consulted. The body acceleration depends on the road conditions and the chosen controller. But it is independent of the energy supply before entering the section. Further we must remember that the sections of the route differs in their length $l_{i}$. This considerations lead to the following evaluation function for a route with deterministic road conditions:

$$
f_{i}\left(x_{i}\right)=\sigma\left(\ddot{z}_{b}\left(d_{i}\left(x_{i}, \mathrm{sc}_{i}\right), \mathbf{s c}_{i}\right)\right) \frac{l_{i}}{\sum_{j=1}^{N} l_{j}} \forall i=1 \ldots N .
$$

We minimize the objective function because a small value of $f_{i}$ connotes a high driving comfort. Since the road conditions are uncertain we consider the three following evaluation functions average-case, worst-case, best-case:

$$
\begin{gathered}
f_{i}^{A C}\left(x_{i}\right)=E\left[\sigma\left(\ddot{z}_{b}\left(d_{i}\left(x_{i}, \mathrm{sc}_{i}\right), \mathrm{sc}_{i}\right)\right)\right] \frac{l_{i}}{\sum_{j=1}^{N} l_{j}} \forall i=1 \ldots N, \\
f_{i}^{B C}\left(x_{i}\right)=\min _{x_{i} \in X_{i}\left(z_{i-1}\right)}\left\{\sigma\left(\ddot{z}_{b}\left(d_{i}\left(x_{i}, \mathrm{sc}_{i}\right), \mathrm{sc}_{i}\right)\right)\right\} \frac{l_{i}}{\sum_{j=1}^{N} l_{j}} \quad \forall i=1 \ldots N, \\
f_{i}^{W C}\left(x_{i}\right)=\max _{x_{i} \in X_{i}\left(z_{i-1}\right)}\left\{\sigma\left(\ddot{z}_{b}\left(d_{i}\left(x_{i}, \mathrm{sc}_{i}\right), \mathrm{sc}_{i}\right)\right)\right\} \frac{l_{i}}{\sum_{j=1}^{N} l_{j}} \quad \forall i=1 \ldots N .
\end{gathered}
$$

The runtime of the DP is mainly determined by the number of considered states and the evaluation of $d_{i}\left(x_{i}, \mathrm{sc}_{i}\right)$ and $f_{i}\left(x_{i}\right)$. Since $d_{i}$ and $f_{i}$ are independent of $z_{i-1}$ and $z_{i}$ they have only be evaluated ones for every possible $x_{i}$. In the modified version of the DP the number $M$ of evaluations is given by:

$$
\begin{aligned}
M & =\sum_{i=1}^{N} \frac{\frac{\hat{e}_{i-1, \max }}{E}\left(\frac{\hat{e}_{i-1, \max }}{E}+1\right)}{2}+\frac{\hat{e}_{i, \max }}{E}\left(\frac{\hat{e}_{i-1, \max }}{E}-\frac{\hat{e}_{i, \max }}{E}\right) \\
& =\sum_{i=1}^{N} \frac{\hat{e}_{i-1, \max }^{2}}{2 E^{2}}+\frac{\hat{e}_{i-1, \max }}{2 E}+\frac{\hat{e}_{i-1, \max } \hat{e}_{i, \max }}{E^{2}}-\frac{\hat{e}_{i, \max }^{2}}{2 E^{2}}
\end{aligned}
$$

The calculated solution is robust against all considered road-scenarios. In case a sectionscenario $\left(\Phi_{0}, \omega, v\right)$ occurs which was not taken into account in the DP the energy consumption on the section will differ from the one in the solution. This leads to a different state respectively remaining energy supply after this section. Due to the optimality principle of Bellman one can change to the solution which is optimal based on the arising state. 


\section{BENCHMARKS AND EXAMPLES}

The benchmarks where computed on a computer with 16 GB RAM and an AMD Phenon II X6 1100T processor. We used a linearised model of the AADS [7] with a Skyhook control. The used damping constants are $5000 \mathrm{~N} \mathrm{~s} \mathrm{~m}^{-1}, 4500 \mathrm{Ns} \mathrm{m}^{-1}, 4000 \mathrm{~N} \mathrm{~s} \mathrm{~m}^{-1}, 3500 \mathrm{~N} \mathrm{~s} \mathrm{~m}^{-1}$, $3000 \mathrm{~N} \mathrm{~s} \mathrm{~m}^{-1}, 2500 \mathrm{~N} \mathrm{~s} \mathrm{~m}^{-1}, 2000 \mathrm{~N} \mathrm{~s} \mathrm{~m}^{-1}, 1500 \mathrm{Ns} \mathrm{m}^{-1}, 1000 \mathrm{Ns} \mathrm{m}^{-1}, 500 \mathrm{~N} \mathrm{~s} \mathrm{~m}^{-1}$ and 0 $\mathrm{N} \mathrm{s} \mathrm{m}^{-1}$. The routes are given by a sequence of roads with road conditions. For every road we use deviations $\Delta \Phi_{0}, \Delta \omega$ and $\Delta v$ to generate the section-scenarios so that one gets three possible value for $\Phi_{0}, \omega$ and $v$. For every section one has 27 section-scenarios and for a route with $N$ sections one has $27^{N}$ route-scenarios. We consider 6 different routes. For detailed information about sections and road conditions see appendix.

- route A contains just autobahn sections and the velocity is $130 \mathrm{~km} / \mathrm{h}$.

- route $\mathbf{B}$ contains just federal road sections and the velocity varies between $95 \mathrm{~km} / \mathrm{h}$ and $110 \mathrm{~km} / \mathrm{h}$.

- route $\mathbf{C}$ is a route from Darmstadt to Ulm (Germany).

- route $\mathbf{D}$ is a route with good road conditions and a high velocity.

- route $\mathbf{E}$ is a route from Frankfurt to Darmstadt (Germany).

- route $\mathbf{F}$ is the route of a commuter which contains only federal roads.

In order to investigate the runtime of the implemented DP we vary the number of road sections $N$ and the energy-step size $E$. This leads to a variation of the steps of the DP and the number of the states in every single step.

Figure 3 shows the runtime over the energy steps. We used the energy-step $5 \mathrm{~J}, 10 \mathrm{~J}, 50$ J, $100 \mathrm{~J}, 500 \mathrm{~J}, 1000 \mathrm{~J}, 5000 \mathrm{~J}, 10000 \mathrm{~J}$. As we expected the runtime behaves like $f(E)=$ $1 / E^{2}+1 / E$ (cf. Fig. 3 ) for small values of $E$. For small values of $E$ respectively a large number of sub-problems the runtime is asymptotically like $f(E)$.

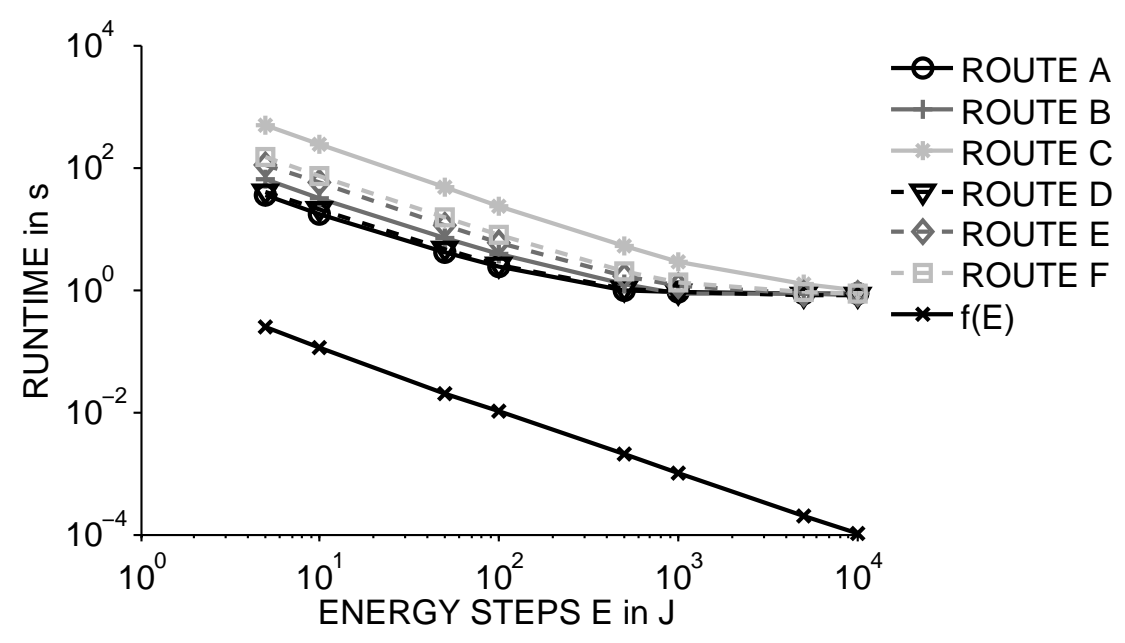

Figure 3: Runtime and function value over the energy steps for all considered routes with axes in a logarithmic scale. 
Figure 4 shows the runtime over the number of route sections for $E=10 \mathrm{~J}$. We generate routes with different numbers of sections, by dividing the section of the considered routes into subsections. As expected the runtime increases linearly with the number of route sections.

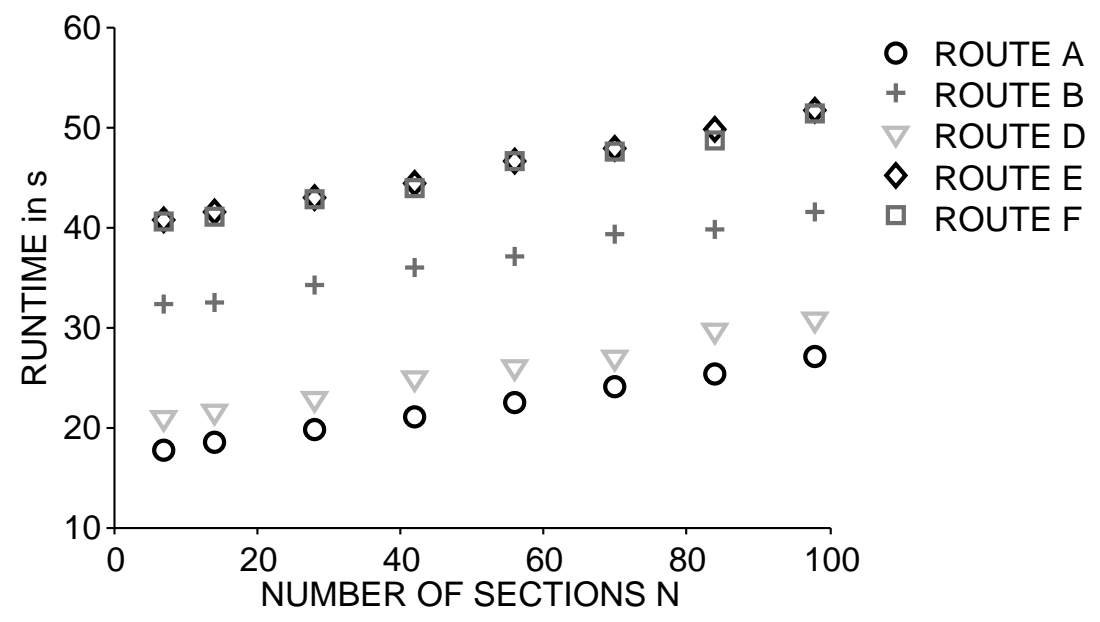

Figure 4: Runtime over the number of route section for all considered routes.

After investigating the runtime we want to have a look at the solution for the route $\mathrm{E}$ for $E=$ $10 \mathrm{~J}$. Figure 5 shows the objective values for all initial states and three evaluation functions. For the total energy supply for the route of $2.1 \mathrm{~kJ}$ the objective values are $\mathrm{BC}=0.275 \mathrm{~m} / \mathrm{s}^{2}$,

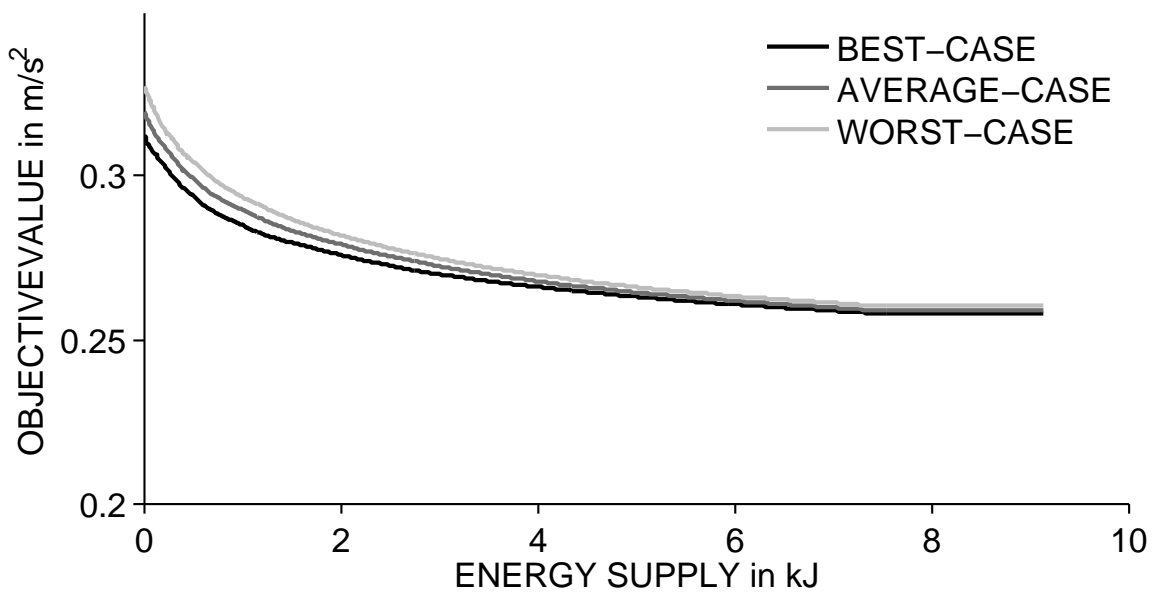

Figure 5: Objective values over the energy supply for route E and an energy step of $10 \mathrm{~J}$.

$\mathrm{AC}=0.278 \mathrm{~m} / \mathrm{s}^{2}$ and $\mathrm{WC}=0.281 \mathrm{~m} / \mathrm{s}^{2}$. It is possible to halve the energy supply with a lose of $3.3 \%, 3.9 \%$ and $4.2 \%$ of comfort in the best-, average- and worst-case.

\section{CONCLUSIONS}

- To resolve the conflict between driving safety and driving comfort active spring damper systems are developed. Since auxiliary power is used for the adaptive control another 
challenge is to handle a limited energy supply while the road conditions are uncertain.

- We compute a operating strategy for the AADS which is robust for all road-scenarios and leads to maximal comfort.

- Discretizeing the energy supply enables us to use DP to solve the problem in reasonable time.

- To relate an increase of demanded energy we use a set of initial energy supplies instead of one.

- The runtime of the DP mainly depends on the calculation of the evaluation function and the section-scenario depending controller. Since $d_{i}$ and $f_{i}$ are independent of $z_{i-1}$ and $z_{i}$ they only need to be evaluated once for every possible $x_{i}$.

- The employed damping constant depends on the occurring section-scenario and the energy supply before entering the section

- In a next step we wave the condition that the road condition get deterministic when entering the section. We will develop a DP which calculates one single damping constant for every section.

\section{ACKNOWLEDGEMENT}

The authors like to thank the German Research Foundation DFG for funding this research within the Collaborative Research Center (SFB) - "Control of Uncertainty in Load-Carrying Structures in Mechanical Engineering".

\section{REFERENCES}

[1] M. Mitschke, H. Wallentowitz, Dynamik der Kraftfahrzeuge. Springer, 2004.

[2] P. Hedrich, F.J. Cloos, J. Würtenberger, P. F. Pelz, Comparison of a New Passive and Active Technology for Vibration Reduction of a Vehicle under Uncertain Load. Applied Mechanics and Materials, 807 pp 57-66, 2015.

[3] B. Heißing, M. Ersoy, Chassis Handbook. Fundamentals, Driving dynamics, Components. Vieweg Teubner, 2011.

[4] H. Braun, Untersuchung von Fahrbahnunebenheiten und Anwendung der Ergebnisse. Dissertation, TU Braunschweig, 1969.

[5] D. Karnopp, Active damping in road vehicle suspension systems Vehicle System Dynamics 12.6 pp 291-311, 1983.

[6] W. Domschke, A. Drexl, Einführung in Operations Research, Vol. 5. Springer, 2005.

[7] T. Bedarff, P. Hedrich, P. F. Pelz, Design of an Active Air Spring Damper. 9. IFK Proceedings Vol.3, 2014. 


\section{APPENDIX}

\begin{tabular}{cccccccc}
\hline$i$ & $l_{i}$ in $\mathrm{km}$ & $\Phi_{0}$ in 1 & $\Delta \Phi_{0}$ in 1 & $\omega$ in $\mathrm{cm}^{3}$ & $\Delta \omega$ in $\mathrm{cm}^{3}$ & $v$ in $\mathrm{m} \mathrm{s}^{-1}$ & $\Delta v$ in $\mathrm{m} \mathrm{s}^{-1}$ \\
\hline 0 & 5.00 & 0.50 & 0.02 & 2.30 & 0.10 & 36.11 & 0.06 \\
1 & 2.00 & 1.00 & 0.07 & 1.50 & 0.06 & 36.11 & 0.11 \\
2 & 2.35 & 1.10 & 0.05 & 1.90 & 0.11 & 36.11 & 0.30 \\
3 & 1.60 & 2.00 & 0.10 & 2.00 & 0.30 & 36.11 & 0.07 \\
4 & 3.20 & 0.90 & 0.06 & 2.60 & 0.07 & 36.11 & 0.09 \\
5 & 2.80 & 1.00 & 0.11 & 2.30 & 0.05 & 36.11 & 0.10 \\
6 & 4.00 & 1.90 & 0.30 & 2.20 & 0.03 & 36.11 & 0.04 \\
\hline
\end{tabular}

Table 2: route A contains just autobahn sections an the velocity is $\mathrm{km} / \mathrm{h}$.

\begin{tabular}{cccccccc}
\hline$i$ & $l_{i}$ in $\mathrm{km}$ & $\Phi_{0}$ in 1 & $\Delta \Phi_{0}$ in 1 & $\omega$ in $\mathrm{cm}^{3}$ & $\Delta \omega$ in $\mathrm{cm}^{3}$ & $v$ in $\mathrm{m} \mathrm{s}^{-1}$ & $\Delta v$ in $\mathrm{m} \mathrm{s}^{-1}$ \\
\hline 0 & 2.00 & 1.30 & 0.02 & 2.10 & 0.10 & 27.78 & 0.06 \\
1 & 2.00 & 2.00 & 0.07 & 1.70 & 0.06 & 30.56 & 0.11 \\
2 & 2.35 & 2.10 & 0.05 & 2.90 & 0.11 & 27.78 & 0.30 \\
3 & 1.60 & 2.00 & 0.10 & 2.00 & 0.30 & 27.78 & 0.07 \\
4 & 3.20 & 1.90 & 0.06 & 2.60 & 0.07 & 29.17 & 0.09 \\
5 & 2.80 & 4.00 & 0.11 & 2.30 & 0.05 & 26.39 & 0.10 \\
6 & 2.00 & 6.00 & 0.30 & 2.60 & 0.03 & 27.78 & 0.04
\end{tabular}

Table 3: route B contains just autobahn sections an the velocity varies between $95 \mathrm{~km} / \mathrm{h}$ and $110 \mathrm{~km} / \mathrm{h}$.

\begin{tabular}{cccccccc}
\hline$i$ & $l_{i}$ in $\mathrm{km}$ & $\Phi_{0}$ in 1 & $\Delta \Phi_{0}$ in 1 & $\omega$ in $\mathrm{cm}^{3}$ & $\Delta \omega$ in $\mathrm{cm}^{3}$ & $v$ in $\mathrm{m} \mathrm{s}^{-1}$ & $\Delta v$ in $\mathrm{m} \mathrm{s}^{-1}$ \\
\hline 0 & 0.42 & 12.50 & 0.02 & 2.50 & 0.10 & 8.33 & 0.06 \\
1 & 2.58 & 9.00 & 0.07 & 2.30 & 0.06 & 13.89 & 0.11 \\
2 & 1.95 & 2.30 & 0.05 & 2.40 & 0.11 & 27.78 & 0.30 \\
3 & 67.91 & 3.00 & 0.10 & 2.00 & 0.30 & 33.33 & 0.07 \\
4 & 186.00 & 1.00 & 0.06 & 2.20 & 0.07 & 36.11 & 0.09 \\
5 & 4.43 & 3.40 & 0.11 & 2.10 & 0.05 & 22.22 & 0.10 \\
6 & 0.28 & 8.00 & 0.30 & 2.60 & 0.03 & 8.33 & 0.04 \\
\hline
\end{tabular}

Table 4: route $\mathrm{C}$ is a route from Darmstad to Ulm (Germany). 


\begin{tabular}{cccccccc}
\hline$i$ & $l_{i}$ in $\mathrm{km}$ & $\Phi_{0}$ in 1 & $\Delta \Phi_{0}$ in 1 & $\omega$ in $\mathrm{cm}^{3}$ & $\Delta \omega$ in $\mathrm{cm}^{3}$ & $v$ in $\mathrm{m} \mathrm{s}^{-1}$ & $\Delta v$ in $\mathrm{m} \mathrm{s}^{-1}$ \\
\hline 0 & 2.00 & 3.00 & 0.02 & 2.60 & 0.10 & 33.33 & 0.06 \\
1 & 2.00 & 1.00 & 0.07 & 1.50 & 0.06 & 36.11 & 0.11 \\
2 & 2.35 & 1.10 & 0.05 & 1.90 & 0.11 & 41.67 & 0.30 \\
3 & 1.60 & 2.00 & 0.10 & 2.00 & 0.30 & 36.11 & 0.07 \\
4 & 3.20 & 0.90 & 0.06 & 2.60 & 0.07 & 41.67 & 0.09 \\
5 & 2.80 & 1.00 & 0.11 & 2.30 & 0.05 & 36.11 & 0.10 \\
6 & 2.00 & 12.00 & 0.30 & 2.40 & 0.03 & 22.22 & 0.04 \\
\hline
\end{tabular}

Table 5: route $\mathrm{D}$ is a route with good road conditions and a high velocity.

\begin{tabular}{cccccccc}
\hline$i$ & $l_{i}$ in $\mathrm{km}$ & $\Phi_{0}$ in 1 & $\Delta \Phi_{0}$ in 1 & $\omega$ in $\mathrm{cm}^{3}$ & $\Delta \omega$ in $\mathrm{cm}^{3}$ & $v$ in $\mathrm{m} \mathrm{s}^{-1}$ & $\Delta v$ in $\mathrm{m} \mathrm{s}^{-1}$ \\
\hline 0 & 0.28 & 12.20 & 0.02 & 2.40 & 0.10 & 8.33 & 0.06 \\
1 & 5.90 & 8.00 & 0.07 & 2.30 & 0.06 & 13.89 & 0.11 \\
2 & 1.35 & 2.10 & 0.05 & 2.30 & 0.11 & 27.78 & 0.30 \\
3 & 20.60 & 1.00 & 0.10 & 2.00 & 0.30 & 36.11 & 0.07 \\
4 & 3.20 & 2.30 & 0.06 & 2.20 & 0.07 & 27.78 & 0.09 \\
5 & 1.75 & 9.00 & 0.11 & 2.40 & 0.05 & 13.89 & 0.10 \\
6 & 0.70 & 10.00 & 0.30 & 2.30 & 0.03 & 8.33 & 0.04 \\
\hline
\end{tabular}

Table 6: route E is a route from Frankfurt to Darmstadt (Germany).

\begin{tabular}{cccccccc}
\hline$i$ & $l_{i}$ in $\mathrm{km}$ & $\Phi_{0}$ in 1 & $\Delta \Phi_{0}$ in 1 & $\omega$ in $\mathrm{cm}^{3}$ & $\Delta \omega$ in $\mathrm{cm}^{3}$ & $v$ in $\mathrm{m} \mathrm{s}^{-1}$ & $\Delta v$ in $\mathrm{m} \mathrm{s}^{-1}$ \\
\hline 0 & 1.00 & 1.70 & 0.02 & 2.10 & 0.10 & 8.33 & 0.06 \\
1 & 2.00 & 2.00 & 0.07 & 1.80 & 0.06 & 13.89 & 0.11 \\
2 & 12.35 & 4.90 & 0.05 & 2.30 & 0.11 & 22.22 & 0.30 \\
3 & 5.80 & 5.30 & 0.10 & 2.40 & 0.30 & 25.00 & 0.07 \\
4 & 4.20 & 2.90 & 0.06 & 2.60 & 0.07 & 13.89 & 0.09 \\
5 & 0.75 & 4.00 & 0.11 & 2.30 & 0.05 & 8.33 & 0.10 \\
6 & 2.00 & 2.00 & 0.30 & 2.60 & 0.03 & 8.33 & 0.04 \\
\hline
\end{tabular}

Table 7: route $\mathrm{F}$ is the daily route of a commuter which contains only federal roads. 